\section{THE IN VITRO EFFECTS OF 5-AZACITIDINE ON THE IMMUNOPHENOTYPE OF MONOCYTE-DERIVED DENDRITIC CELLS FROM PATIENTS WITH HIGHER-RISK MYELODYSPLASTIC SYNDROMES}

${ }^{1}$ Randy Tsai*, ${ }^{2}$ Hannah Fields, 'Xinlian Zhang, ${ }^{3}$ Valentina Ferrari, 'Soo Park, 'Rafael Bejar, ${ }^{4}$ Tiffany Tanaka. ${ }^{1}$ UC San Diego, San Diego, CA, United States; ${ }^{2}$ ImmunoScape, San Diego, CA, United States; ${ }^{3}$ Humanitas University, Milan, Italy; ${ }^{4}$ UC San Diego Moores Cancer Center, San Diego, United States

Background Myelodysplastic syndromes (MDS) are the most common acquired cause of bone marrow failure. Though DNA hypomethylating agents (HMAs) such as 5-Azacitidine (5-Aza) may increase survival of patients with higher-risk MDS, their mechanistic effects on hematopoiesis and immune cell function remain unclear. Using whole exome sequencing analysis, we previously identified MDS-related mutations within monocyte-derived dendritic cells (moDCs) from patients with higher-risk MDS. Here we examine the effect of 5-Aza on the phenotype of moDCs from the same cohort of patients with higher-risk MDS.

Methods Purified CD14+ cells were magnetically isolated from peripheral blood mononuclear cells from 6 patients with IPSS-R Intermediate/High/Very High-risk MDS (herein collectively referred to as higher-risk MDS). Cells were cultured in complete medium with IL-4 (800 U/mL) and GM-CSF (1200 $\mathrm{U} / \mathrm{mL}$ ) for 5 days. Freshly prepared 5-Aza or dimethylsulfoxide (DMSO) vehicle was added to cultures every 24 hours for a total of three $1 \mu \mathrm{M}$ doses starting on Day 1 . Immature moDCs were then stimulated with poly(I:C) $(20 \mathrm{ng} / \mathrm{mL})$, IL$1 \beta(25 \mathrm{ng} / \mathrm{mL})$, IFN- $\alpha(3000 \mathrm{U} / \mathrm{mL})$, IFN $-\gamma(1000 \mathrm{U} / \mathrm{mL})$, and TNF- $\alpha(50 \mathrm{ng} / \mathrm{mL})$ for 48 hours to generate moDCs. Flow cytometry analyses were performed with Guava easyCyte 8HT before and after addition of maturation cocktail.

Results Based on trypan blue staining, in vitro addition of 5Aza to CD14+ cells from 6 patients with higher-risk MDS did not result in a significant reduction in the percentage of cell survival on Day 5 and Day 7 in culture (figure 1a, $\mathrm{p}=0.8765$ and $\mathrm{p}=0.7109$, respectively). Treatment with 5 -Aza significantly reduced the percentage of CD14-CD209+ moDCs on Day 7 following the addition of maturation cocktail (figure $1 \mathrm{~b}, \mathrm{p}<0.0001)$. Flow cytometry assessment showed comparable expression of common maturation and co-stimulatory markers such as CD80, CD83, CD86, HLA-DR, CD209, CD141, CD40, and CCR7 between 5-Aza and DMSO-treated immature moDCs on Day 5 (figure 1c). Similarly, 5-Aza treatment had no significant effect on marker expression on mature moDCs generated with maturation cocktail on Day 7 .

Conclusions There was no significant difference in maturation and co-stimulatory marker expression of immature and mature moDCs from patients with higher-risk MDS following in vitro treatment with 5-Aza. Though recent studies have identified important immunoregulatory effects of 5-Aza, functional changes that may occur within the dendritic cell population are not fully understood. Further studies are planned, including cytokine analyses and transcriptome sequencing of mature moDCs, and may help elucidate the immunological mechanisms underlying the therapeutic effects of 5-Aza in patients with higher-risk MDS.

Ethics Approval The study is being conducted as per the Declaration of Helsinki and was approved by the University of California San Diego Institutional Review Board (\#161345) and registered with ClinicalTrials.gov (NCT02667093). All patients were provided written informed consent.
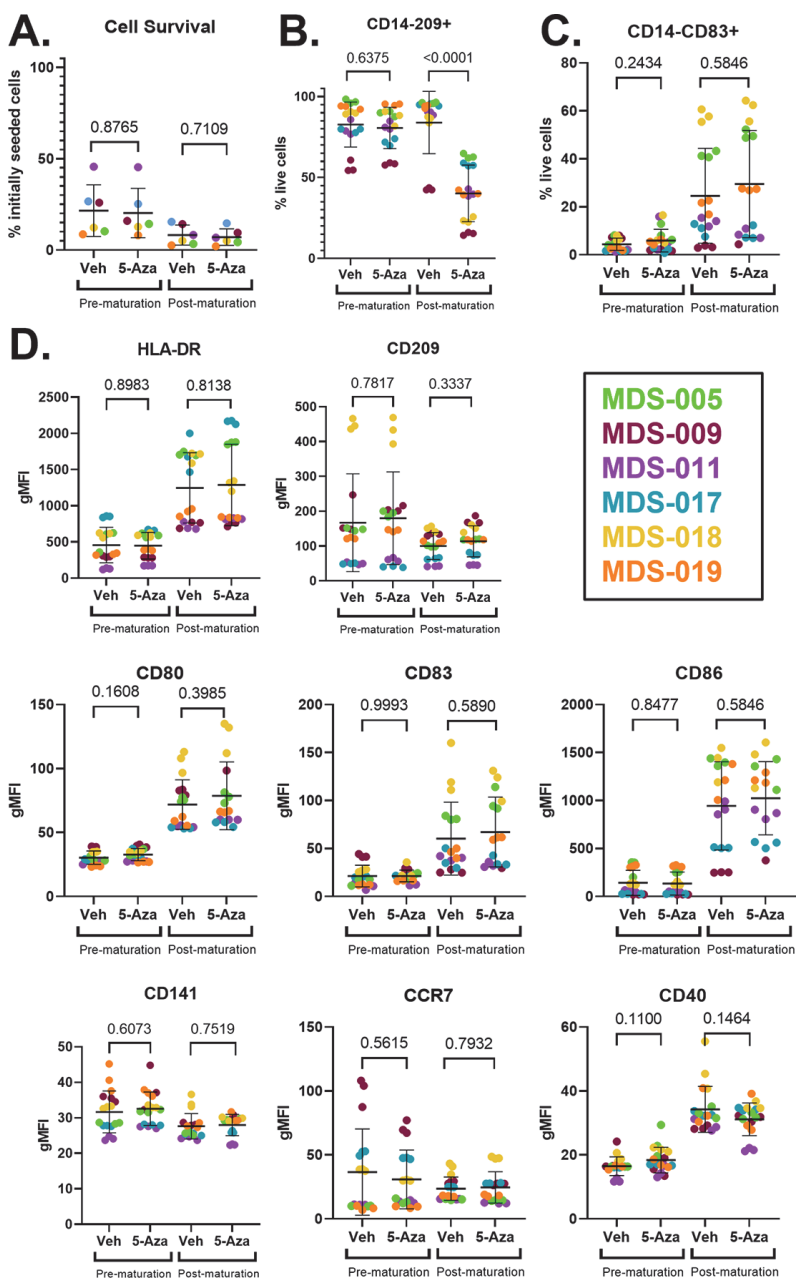

Abstract 684 Figure 1 5-Aza and DMSO vehicle-treated moDCs from patients with higher-risk MDS were evaluated for phenotypic markers before and after stimulation with maturation cocktail. Purified CD14+ cells were magnetically isolated from PBMC from 6 higher-risk MDS patients and cultured with IL- 4 and GM-CSF for 5 days followed by addition of poly(I:C), IL-1 $\beta$, IFN- $\alpha$, IFN- $\gamma$, and TNF- $\alpha$ for 48 hours at $37^{\circ} \mathrm{C}$ in a $5 \%$ CO2 incubator. Freshly prepared 5-Aza or DMSO vehicle was added to cultures every 24 hours for a total of three $1 \mu \mathrm{M}$ doses starting on Day 1. (A) Cultured cells were stained with trypan blue to determine the percentage of cell survival on Day 5 and Day 7 in culture. (B) Treatment with 5-Aza significantly reduced the percentage of CD14-CD209+ moDCs on Day 7 following addition of maturation cocktail $(p<0.0001)$. (C) The percentage of CD14-CD83+ cells is comparable between 5-Aza and vehicle-treated immature moDCs on Day 5 and mature moDCs on Day $7(p=0.2434$ and $p=0.5846$, respectively). (D) Cultured cells were stained with fluorochromeconjugated antibodies to determine the expression of common maturation and co-stimulatory markers using flow cytometry. Cells were gated on CD14-CD11c+ to distinguish moDCs, and scatterplots represent the geometric mean fluorescence intensity (gMFI) of marker expression pre- and post-maturation. Individual dots represent one of three experimental replicates performed for the 6 higher-risk MDS patient samples. Each dot is labeled by MDS patient sample. Statistical analysis was performed by Welch's t-test using GraphPad Prism.

http://dx.doi.org/10.1136/jitc-2021-SITC2021.684 